\title{
Robotic Resection of a Recurrent Pediatric Lipoblastoma
}

\author{
Cory N. Criss MD ${ }^{1}$, Christa Grant MD ${ }^{1}$, Matthew W. Ralls MD', \\ James D. Geiger MD
}

\author{
Section of Pediatric Surgery, C.S. Mott Children's Hospital, University of Michigan, Ann \\ Arbor, MI, USA ${ }^{1}$ \\ Corresponding Author: Cory N. Criss, MD \\ Email: cocriss@med.umich.edu \\ Tel: $734-764-6482$ \\ Fax: 734-232-8667
}

Short Running Title: Robotic Resection of Pediatric Lipoblastoma

\begin{abstract}
This case demonstrates successful resection of a rare, recurrent presacral-pelvic lipoblastoma in a 19-year-old female. Due to the anatomical location of the mass and its proximity to vital structures, the robotic approach allowed for both optimal visualization and effective debulking of the mass. Furthermore, with the utilization of the articulating laparoscopic camera, key visualization of the posterior lateral pelvis was possible. Utilizing a wide breadth of technologies and resources is essential to broadening the surgical armamentarium and achieving resectability in otherwise challenging cases.
\end{abstract}

Keywords: lipoblastoma, pediatric surgery, robotic resection

This is the author manuscript accepted for publication and has undergone full peer review but has not been through the copyediting, typesetting, pagination and proofreading process, which may lead to differences between this version and the Version of Record. Please cite this article as doi: 10.1111 /ases.12493

This article is protected by copyright. All rights reserved. 


\section{Introduction}

Arising from embryonic fat tissue, lipoblastomas are rare pediatric tumors initially discovered in 1926 by Jaffe et al. and subsequently described in 1958 by Vellios et al.[1, 2] While these tumors are benign, they have a tendency to grow rapidly, impinging on and enveloping surrounding structures, leading to symptoms. They are most commonly associated with regions of immature adipose tissue in the soft tissues of the trunk and extremities, but have also been shown to arise in the mediastinum, anterior chest, abdomen, neck, face, rectal area, and pre-sacral region.[3] While there are only case reports and case series in literature, the mainstay of treatment relies on surgical excision unless extreme circumstances are present.[4] Surgical resection can be curative, but there remains a $12-25 \%$ recurrence rate. $[5,6]$ The optimal approach to surgical excision is not well studied, with very limited literature on minimally invasive approaches.

The da Vinci Surgical System (Intuitive Surgical, Sunnyvale, CA) has been utilized over the years in various sub-specialties operating in the pelvis such as gynecology, colorectal surgery, and urology.[7] By providing multiple degrees of freedom in a limited space, the characteristics of the robot are optimal for deep pelvic procedures, such as the one presented here. [8] Here, we present a rare case of recurrent lipoblastoma in a 19-year-old female with persistent leg and buttock pain as well as pelvic and gluteal muscle atrophy. Due to the location of the mass on imaging, the decision to utilize a robotic assisted approach for resection was made. By combining the unique characteristics of the robot in addition to laparoscopic image guidance with the Olympus EndoEye (Olympus, Center Valley, PA), an otherwise difficult case was successfully completed using a minimally invasive approach. This case highlights the true advantages of the robot and emphasizes the value of the additional advanced imaging 
technologies (EndoEye, MRI and laparoscopic ultrasound). Utilization of these technologies together ultimately led to a successful outcome.

\section{Case Presentation}

\section{History of Present Illness}

A 19-year-old female who was referred to the multidisciplinary tumor oncology clinic for evaluation of a left sciatic mass. The patient had a history of mass excision at two years of age with confirmed lipobastoma on pathology. She was followed for 10 years post-excision with serial imaging, and discharged with no signs of recurrence. For the 6 months prior to presentation, she noted increasing left buttock and thigh pain, with worsening symptoms on knee flexion and foot dorsiflexion. CT and MRI imaging demonstrated a $7.3 \times 8.3 \times 8.9$ multi-lobulated mass in the left presacral and sciatic region, extending laterally along the tissues near the iliac wing, and through the sciatic foramen into the gluteal region (see Figure 1). After multidisciplinary consultation, she was scheduled for excision via a trans-abdominal approach for surgical excision and intraoperative frozen section to confirm diagnosis. If additional posterior resection was required, plastic surgery and neurosurgery was available for further resection.

\section{Laparoscopic Assisted Robotic Resection}

After general anesthesia was obtained and intubation was achieved, the patient was placed in lithotomy with arms tucked to allow an anterior robotic approach. The abdomen was prepped and draped in the normal sterile fashion. After achieving intraabdominal access, ports were placed in the right and left upper quadrants. Utilizing the da Vinci robot (Intuitive Surgical, Sunnyvale, CA), the sigmoid colon and rectum were mobilized laterally, traveling caudally towards the pelvis. The left ureter was identified, and traced down to the pelvis. The left ovary and fallopian tube were mobilized superiorly. The uterus was suspended anteriorly using a silk suture pulled through the abdominal wall. With the rectum retracted laterally, further dissection visualized the tumor bulge deep in the left hemi-pelvis. Using the robot, the peritoneum was opened and the capsule of the mass was exposed. A specimen was sent for frozen section, which demonstrated "myxoid tissue with lipoblasts, consistent with a lipoblastoma". Due to the anatomical location of the mass and limited working space, the robot allowed for 
successful visualization and what was felt to be a gross/near total excision of the pelvic portion of the mass. The mass had both solid and gelatinous components, requiring a piecemeal excision and suction for removal. The dissection and removal was carried along the path of the mass, which traveled along the lateral portions of the pelvis extending caudally to the obturator muscle.

After substantial debulking of the mass anteriorly, an MRI was obtained during the operation to confirm near total debulking (see Figure 2, Image A). This demonstrated a potential residual intra-pelvic component along the lateral pelvic sidewall. An intraoperative ultrasound was also utilized at this time, which identified an additional fatty lesion in this area. To obtain better visualization, it was decided to use the Olympus EndoEye (Olympus, Center Valley, PA) (Figure 2, Image C). Utilizing this flexible imaging system, the supero-lateral component was visualized beneath the nerve roots posteriorly, in the area corresponding to the ultrasound image; however the area could not be reached with standard straight laparoscopic instruments. Once again, utilizing the dexterity and articulation of the da Vinci robot (Intuitive Surgical, Sunnyvale, CA), a large amount of tumor from the left posterior component was successfully removed piecemeal (see Figure 2, Image B). After hemostasis was obtained, the abdomen was desufflated and ports were closed at the fascia and skin levels. With a total procedure time of 16 hours, the patient was extubated successfully and taken to the PACU in stable condition.

\section{Postoperative Course}

Postoperatively, the patient's pain was adequately controlled and she was advanced to a regular diet postoperative day 2. She was evaluated by physical therapy and was noted to have residual numbness but improved pain along her left leg and buttock. She received a postoperatively CT and MRI to evaluate post-resection, which demonstrated post-surgical changes and successful debulking of the mass with limited evidence of residual tissue. After having bowel movements, tolerating a regular, and adequate pain control, she was discharged on postoperative day 4 with plans for followup and serial imaging.

\section{Discussion}




\section{Criss 5}

This case demonstrates successful resection of a rare, recurrent presacral-pelvic lipoblastoma in a 19-year-old female. Due to the anatomical location of the mass and its proximity to vital structures, the robotic approach allowed for both optimal visualization and effective debulking of the mass. Furthermore, with the utilization of the Olympus EndoEye (Olympus, Center Valley, PA), an articulating laparoscopic camera, key visualization of the posterior lateral pelvis was possible.

While there is limited literature on the optimal approach to lipoblastoma resections due to their rarity, this case demonstrates a potential use of multiple resources, including the da Vinci robot (Intuitive Surgical, Sunnyvale, CA) in a technically challenging case. The robotic system allows for intuitive control over the instruments with the ability to articulate based on the seven degrees of freedom. This, in addition to the 3-D imaging capabilities, facilitates an experience and maneuverability similar to open procedures. Furthermore, the added ergonomic support of the surgical system helps to facilitate a more comfortable surgeon experience for prolonged dissections in the pelvis, such as the one presented here. Despite these proposed advantages, there is a great deal of criticism over the clinical evidence supporting the robot for commonly performed procedures compared to conventional laparoscopy. However, its unique abilities during pelvic surgery have been well described in the past.

The long-term prognosis of patients with lipoblastoma, even recurrent tumors, remains excellent. There is no evidence of malignant degeneration in the literature to date; however, local recurrence remains a concern, with rates quoted as $12-25 \%$ in some limited small case series and reports.[9] Interestingly, most recurrences have been noted to occur within 2 years of resection, which is in stark contrast to our patient, who presented almost 17 years later. [10] Even so, authors recommend close follow-up to at least 5 years post-resection to monitor closely for recurrence.

As technology continues to advance at rapid pace, the opportunities for surgeons to expand their operative abilities also increases. While robotic surgery is commonly practiced among many individuals, including pediatric surgeons, utilizing additional technologies such as the articulating laparoscopic camera is just one additional adjunct to aid in the operation. Here, we highlight successful resection of an anatomically challenging mass in a pediatric patient through an entirely minimally invasive approach.

This case demonstrates successful resection of a recurrent lipoblastoma utilizing the unique characteristics of the robot for an anatomically difficult resection. Utilizing a wide breadth of technologies and resources is essential to broadening the surgical armamentarium and achieving resectability in otherwise challenging cases. 


\section{Criss 6}

Conflicts of Interest: The authors have no disclosures related to this subject.

\section{Reference List}

1. Vellios, F., J. Baez, and H.B. Shumacker, Lipoblastomatosis: a tumor of fetal fat different from hibernoma; report of a case, with observations on the embryogenesis of human adipose tissue. Am J Pathol, 1958. 34(6): p. 1149-59.

2. O'Donnell, K.A., Caty MG, Allen JE, et al., Lipoblastoma: better termed infantile lipoma? Pediatr Surg Int, 2000. 16(5-6): p. 458-61.

3. Al-Mubarak, G., Bryant AS, Crawford JH, et al., Surgical and Anesthetic Management of a Mediastinal Fatty Tumor: Lipoblastoma. Ann Thorac Surg, 2015. 100(5): p. e97-8.

This article is protected by copyright. All rights reserved. 
4. Mahour, G.H., B.J. Bryan, and H. Isaacs, Jr., Lipoblastoma and lipoblastomatosis--a report of six cases. Surgery, 1988. 104(3): p. 577-9.

5. McVay, M.R., Keller JE, Wagner CW, et al., Surgical management of lipoblastoma. J Pediatr Surg, 2006. 41(6): p. 1067-71.

6. Dilley, A.V.,Patel DL, Hicks MJ, et al., Lipoblastoma: pathophysiology and surgical management. J Pediatr Surg, 2001. 36(1): p. 229-31.

7. Ahmed, K., Khan MS, Vats A, et al., Current status of robotic assisted pelvic surgery and future developments. Int J Surg, 2009. 7(5): p. 431-40.

8. Goldsmith, Z.G, Astroza GM, Wang AJ, et al., Optical performance comparison of deflectable laparoscopes for laparoendoscopic single-site surgery. J Endourol, 2012. 26(10): p. 1340-5.

9. Kerkeni, Y, Sahoun L, Ksia A et al., Lipoblastoma in childhood: about 10 cases. Afr J Paediatr Surg, 2014. 11(1): p. 32-4.

10. Cudnik, R., Efron PA, Chen MK, et al., Mesenteric lipoblastoma: a rare location in children. J Pediatr Surg, 2008. 43(12): p. e5-7.

Figure Legend 1: Images A and B represent 3-D CT scan reconstruction of lipoblastoma (red). Images $\mathrm{C}$ and D demonstrate sagittal (C) and axial (D) CT images of lipoblastoma (white dotted line).

Figure Legend 2: Image A: MRI demonstrates residual lateral component along pelvis (white arrow). Image B: Utilizing the robot for resection of lateral segment (white dotted line), highlighting articulation necessary for accessibility. Image C: View of lateral segment using Olympus EndoEye 
Image 1: Preoperative Imaging

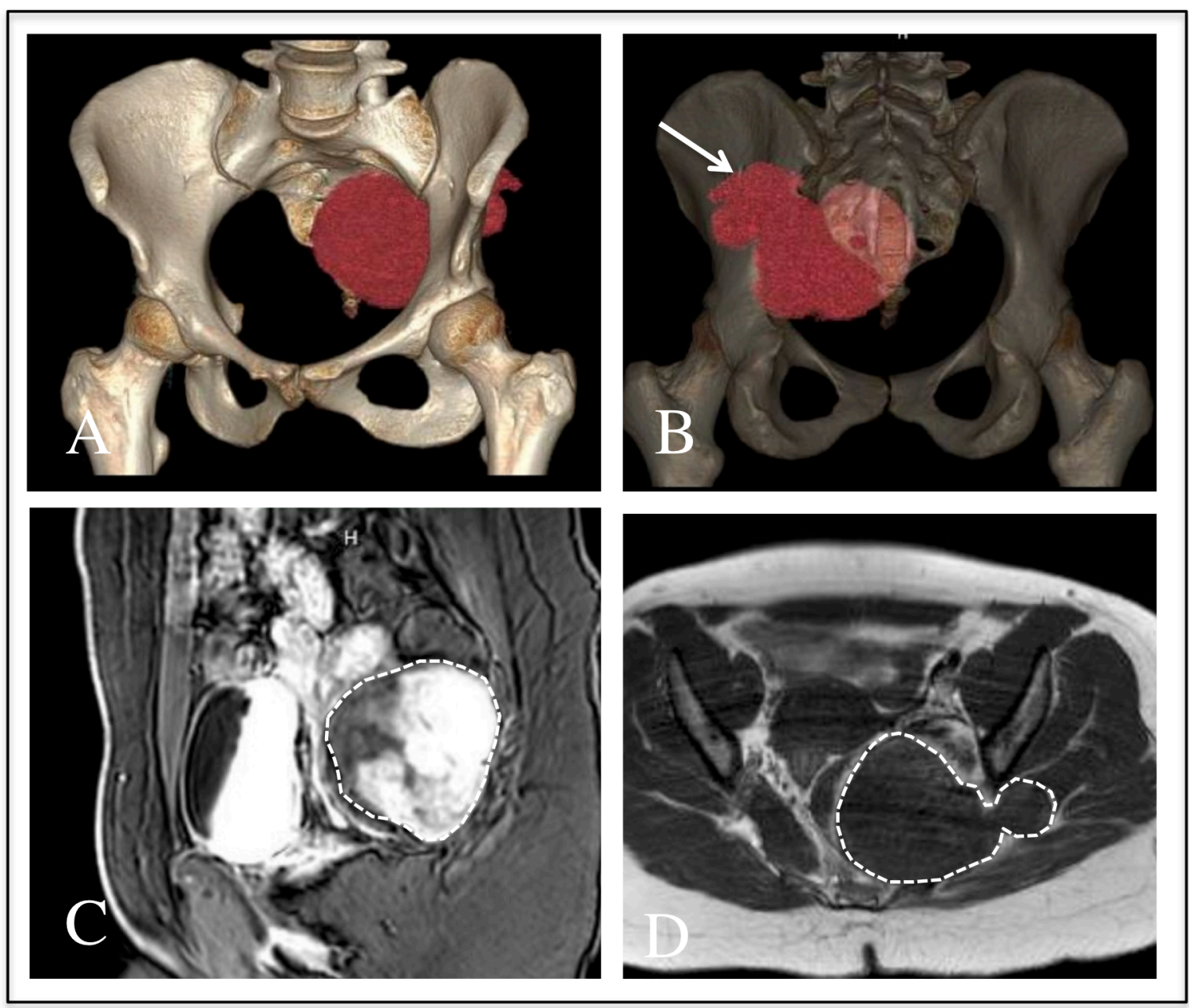

Figure-1.tiff

This article is protected by copyright. All rights reserved. 
Figure 2: Intraoperative Imaging and Robotic Assisted Resection of Lipoblastoma

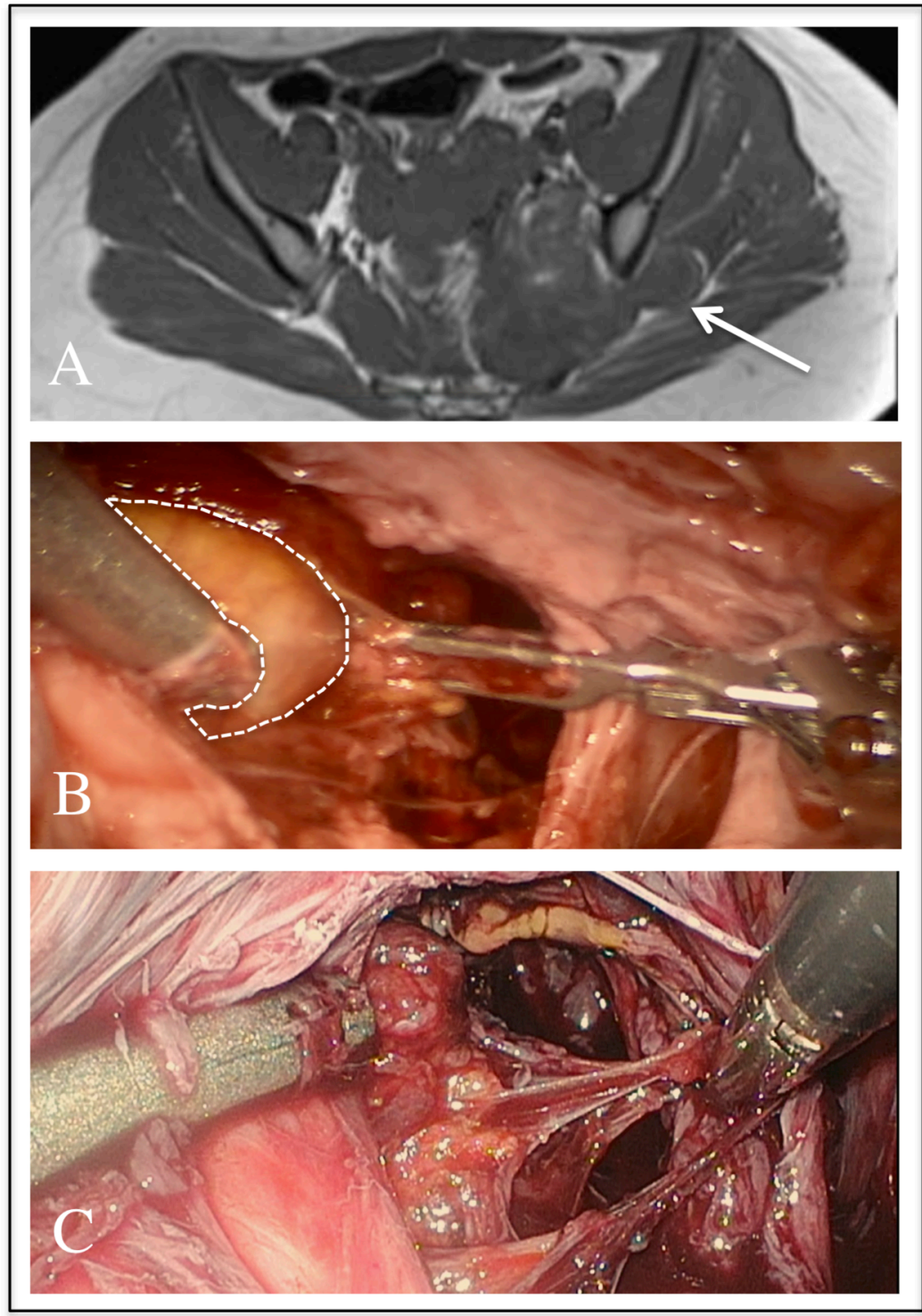

Figure-2.tiff

This article is protected by copyright. All rights reserved. 